\title{
ERGODIC SOLENOIDAL HOMOLOGY: REALIZATION THEOREM
}

\author{
VICENTE MUÑOZ AND RICARDO PÉREZ MARCO
}

\begin{abstract}
We define generalized currents associated with immersions of abstract oriented solenoids with a transversal measure. We realize geometrically the full real homology of a compact manifold with these generalized currents, and more precisely with immersions of minimal uniquely ergodic solenoids. This makes precise and geometric De Rham's realization of the real homology by only using a restricted geometric subclass of currents.
\end{abstract}

\section{INTRODUCTION}

We consider a smooth compact connected oriented manifold $M$ of dimension $n \geq 1$. Any closed oriented submanifold $N \subset M$ of dimension $0 \leq k \leq n$ determines a homology class in $H_{k}(M, \mathbb{Z})$. This homology class in $H_{k}(M, \mathbb{R})$, as dual of De Rham cohomology, is explicitly given by integration of the restriction to $N$ of differential $k$-forms on $M$. Also, any immersion $f: N \rightarrow M$ defines an integer homology class in a similar way by integration of pull-backs of $k$-forms. Unfortunately, because of topological reasons dating back to Thom [Th1] [Th2], not all integer homology classes in $H_{k}(M, \mathbb{Z})$ can be realized in such a way. Geometrically, we can realize any class in $H_{k}(M, \mathbb{Z})$ by topological $k$-chains. The real homology $H_{k}(M, \mathbb{R})$ classes are only realized by formal combinations with real coefficients of $k$-cells. This is not satisfactory for various reasons. In particular, for diverse purposes it is important to have an explicit realization, as geometric as possible, of real homology classes.

The first contribution in this direction came in 1957 from the work of S. Schwartzman Sc. Schwartzman showed how, by a limiting procedure, one-dimensional curves embedded in $M$ can define a real homology class in $H_{1}(M, \mathbb{R})$. More precisely, he proved that this happens for almost all curves solutions to a differential equation admitting an invariant ergodic probability measure. Schwartzman's idea is very natural. It consists on integrating 1-forms over large pieces of the parametrized curve and normalizing this integral by the length of the parametrization. Under suitable conditions, the limit exists and defines an element of the dual of $H^{1}(M, \mathbb{R})$, i.e. an element of $H_{1}(M, \mathbb{R})$. This procedure is equivalent to the more geometric one of closing large pieces of the curve by relatively short closing paths. The closed curve obtained defines an integer homology class. The normalization by the length of the parameter range provides a class in $H_{k}(M, \mathbb{R})$. Under suitable hypothesis, there exists a unique limit in real homology when the pieces exhaust the parametrized curve, and this limit is independent of the closing procedure. In the article [MPM3], we study the different aspects of the Schwartzman procedure, that we extend to higher dimension.

Later in 1975, D. Ruelle and D. Sullivan [RuSu defined, for arbitrary dimension $0 \leq k \leq$ $n$, geometric currents by using oriented $k$-laminations embedded in $M$ and endowed with a

2000 Mathematics Subject Classification. Primary: 37A99. Secondary: 58A25, 57R95, 55N45.

Key words and phrases. Real homology, Ruelle-Sullivan current, solenoid, ergodic theory.

Partially supported through Spanish MEC grant MTM2007-63582. Second author supported by CNRS (UMR 7539). 
transversal measure. They applied their results to stable and unstable laminations of Axiom A diffeomorphisms. In a later article Sullivan [Su1] extended further these results and their applications. The point of view of Ruelle and Sullivan is also based on duality. The observation is that $k$-forms can be integrated on each leaf of the lamination and then all over the lamination using the transversal measure. This makes sense locally in each flow-box, and then it can be extended globally by using a partition of unity. The result only depends on the cohomology class of the $k$-form. In MPM2] we review and extend Ruelle-Sullivan theory.

It is natural to ask whether it is possible to realize every real homology class using a topologically minimal (i.e. all leaves dense) Ruelle-Sullivan current. In order to achieve this goal we must enlarge the class of Ruelle-Sullivan currents by considering immersions of abstract oriented solenoids. We define a $k$-solenoid to be a Hausdorff compact space foliated by $k$ dimensional leaves with finite dimensional transversal structure (see the precise definition in section 2). For these oriented solenoids we can consider $k$-forms that we can integrate provided that we are given a transversal measure invariant by the holonomy group. We define an immersion of a solenoid $S$ into $M$ to be a regular map $f: S \rightarrow M$ that is an immersion in each leaf. If the solenoid $S$ is endowed with a transversal measure $\mu$, then any smooth $k$-form in $M$ can be pulled back to $S$ by $f$ and integrated. The resulting numerical value only depends on the cohomology class of the $k$-form. Therefore we have defined a closed current that we denote by $\left(f, S_{\mu}\right)$ and that we call a generalized current. This gives a homology class $\left[f, S_{\mu}\right] \in H_{k}(M, \mathbb{R})$. Our main result is:

Theorem 1.1. (Realization Theorem) Every real homology class in $H_{k}(M, \mathbb{R})$ can be realized by a generalized current $\left(f, S_{\mu}\right)$ where $S_{\mu}$ is an oriented, minimal, uniquely ergodic solenoid.

Minimal and uniquely ergodic solenoids are defined later on. This result strengths De Rham's realization theorem of homology classes by abstract currents, i.e. forms with coefficients distributions. It is a geometric De Rham's Theorem where the abstract currents are replaced by generalized currents that are geometric objects.

We can ask why we do need to enlarge the class of Ruelle-Sullivan currents. The result does not hold for minimal Ruelle-Sullivan currents due to the following observation from [MPM2] (compare with $[\mathrm{HM}]$ ).

Proposition 1.2. Homology classes with non-zero self-intersection cannot be represented by Ruelle-Sullivan currents with no compact leaves.

Therefore it is not possible to represent a real homology class in $H_{k}(M, \mathbb{R})$ with non-zero self-intersection by a minimal Ruelle-Sullivan current that is not a submanifold. Note that this obstruction only exists when $n-k$ is even. This may be the historical reason behind the lack of results on the representation of an arbitrary homology class by minimal Ruelle-Sullivan currents.

The space of solenoids is large, and we would like to realize the real homology classes by a minimal class of solenoids enjoying good properties. We are first naturally led to topological minimality. As we prove in [MPM2, the spaces of $k$-solenoids is inductive and therefore there are always minimal $k$-solenoids. However, the transversal structure and the holonomy group of minimal solenoids can have a rich structure. In particular, such a solenoid may have many distinct transversal measures, each one yielding a different generalized current for the same immersion $f$. Also when we push Schwartzman ideas beyond 1-homology for some nice classes of solenoids, we see that in general, even when the immersion is an embedding, the generalized 
current does not necessarily coincide with the Schwartzman homology class of the immersion of each leaf (actually not even this Schwartzman class needs to be well defined). Indeed the classical literature lacks of information about the precise relation between Ruelle-Sullivan and Schwartzman currents. One would naturally expect that there is some relation between the generalized currents and the Schwartzman current (if defined) of the leaves of the lamination. We study this problem in MPM3. The main result is that there is such relation for the class of minimal, ergodic solenoids with a trapping region. A solenoid with a trapping region (see definition in section 2) has holonomy group generated by a single map. Then the bridge between generalized currents and Schwartzman currents of the leaves is provided by Birkhoff's ergodic theorem. The main result of [MPM3] is the following.

Theorem 1.3. Let $S_{\mu}$ be a minimal solenoid endowed with an ergodic transversal measure $\mu$ and possessing a trapping region $W$. Let $f: S_{\mu} \rightarrow M$ be an immersion of $S_{\mu}$ into $M$ such that $f(W)$ is contained in a ball of $M$. Then for $\mu$-almost all leaves $l \subset S_{\mu}$, the Schwartzman homology class of $f(l) \subset M$ is well defined and coincides with the homology class $\left[f, S_{\mu}\right]$.

If moreover $S$ is uniquely ergodic, then this happens for all leaves.

(We recall the definition of Schwartzman homology class and trapping region in section 2.)

The solenoids constructed for the proof of the Realization Theorem do satisfy the hypothesis of this theorem and the transversal measure is unique, that is, the solenoids are uniquely ergodic.

\section{Solenoidal Hodge Conjecture.}

The Hodge Conjecture is an statement about the geometric realization of an integral class of pure type $(p, p)$ in a complex (projective) manifold. If we drop the condition of the class being integral, then theorem 1.1]suggests a natural conjecture for real homology classes of pure type as follows.

For a compact Kähler manifold $M$ of complex dimension $n$, a complex immersed solenoid $f: S_{\mu} \rightarrow M$ (that is, a solenoid where the images $f(l)$ of the leaves $l \subset S_{\mu}$ are complex immersed submanifolds), of dimension $k=2(n-p)$, defines a class in $H_{n-p, n-p}(M)=H^{p, p}(M)^{*} \subset$ $H_{k}(M, \mathbb{R})$, as proved in proposition 7.8 of [MPM2]. It is natural to formulate the following conjecture:

Conjecture 1.4. (Solenoidal Hodge Conjecture) Let $M$ be a compact Kähler manifold. Then any class in $H^{p, p}(M)$ is represented by a complex immersed solenoid of dimension $k=$ $2(n-p)$.

Note that the standard Hodge Conjecture is stated for projective complex manifolds, since it fails for Kähler manifolds $[\mathrm{Zu}$. The counterexamples of $[\mathrm{Zu}$ are non-algebraic complex tori. It is easy to see that conjecture 1.4 holds for complex tori (using non-minimal complex solenoids).

Acknowledgements. The authors are grateful to Alberto Candel, Etienne Ghys, Nessim Sibony, Dennis Sullivan and Jaume Amorós for their comments and interest on this work. In particular, Etienne Ghys early pointed out on the impossibility of realization in general of integer homology classes by embedded manifolds.

The first author wishes to acknowledge Universidad Complutense de Madrid and Institute for Advanced Study at Princeton for their hospitality and for providing excellent working conditions. The second author thanks Jean Bourgain and the IAS at Princeton for their hospitality and facilitating the collaboration of both authors. 


\section{Solenoids AND GENERALIZED CURRENTS}

Let us review the main concepts introduced in [MPM2].

Definition 2.1. A $k$-solenoid, where $k \geq 0$, of class $C^{r, s}$, is a compact Hausdorff space endowed with an atlas of flow-boxes $\mathcal{A}=\left\{\left(U_{i}, \varphi_{i}\right)\right\}$,

$$
\varphi_{i}: U_{i} \rightarrow D^{k} \times K\left(U_{i}\right)
$$

where $D^{k}$ is the $k$-dimensional open ball, and $K\left(U_{i}\right) \subset \mathbb{R}^{l}$ is the transversal set of the flow-box. The changes of charts $\varphi_{i j}=\varphi_{i} \circ \varphi_{j}^{-1}$ are of the form

$$
\varphi_{i j}(x, y)=(X(x, y), Y(y))
$$

where $X(x, y)$ is of class $C^{r, s}$ and $Y(y)$ is of class $C^{s}$.

Let $S$ be a $k$-solenoid, and $U \cong D^{k} \times K(U)$ be a flow-box for $S$. The sets $L_{y}=D^{k} \times\{y\}$ are called the (local) leaves of the flow-box. A leaf $l \subset S$ of the solenoid is a connected $k$ dimensional manifold whose intersection with any flow-box is a collection of local leaves. The solenoid is oriented if the leaves are oriented (in a transversally continuous way).

A transversal for $S$ is a subset $T$ which is a finite union of transversals of flow-boxes. Given two local transversals $T_{1}$ and $T_{2}$ and a path contained in a leaf from a point of $T_{1}$ to a point of $T_{2}$, there is a well-defined holonomy map $h: T_{1} \rightarrow T_{2}$. The holonomy maps form a pseudogroup.

A $k$-solenoid $S$ is minimal if it does not contain a proper sub-solenoid. By [MPM2, section 2], minimal sub-solenoids do exist in any solenoid. If $S$ is minimal, then any transversal is a global transversal, i.e., it intersects all leaves. In the special case of an oriented minimal 1-solenoid, the holonomy return map associated to a local transversal,

$$
R_{T}: T \rightarrow T
$$

is known as the Poincaré return map (see [MPM2, Section 4]).

Definition 2.2. Let $S$ be a k-solenoid. A transversal measure $\mu=\left(\mu_{T}\right)$ for $S$ associates to any local transversal $T$ a locally finite measure $\mu_{T}$ supported on $T$, which are invariant by the holonomy pseudogroup, i.e. if $h: T_{1} \rightarrow T_{2}$ is a holonomy map, then $h_{*} \mu_{T_{1}}=\mu_{T_{2}}$.

We denote by $S_{\mu}$ a $k$-solenoid $S$ endowed with a transversal measure $\mu=\left(\mu_{T}\right)$. We refer to $S_{\mu}$ as a measured solenoid. Observe that for any transversal measure $\mu=\left(\mu_{T}\right)$ the scalar multiple $c \mu=\left(c \mu_{T}\right)$, where $c>0$, is also a transversal measure. Notice that there is no natural scalar normalization of transversal measures.

Definition 2.3. (Transverse ergodicity) A transversal measure $\mu=\left(\mu_{T}\right)$ on a solenoid $S$ is ergodic if for any Borel set $A \subset T$ invariant by the pseudo-group of holonomy maps on $T$, we have

$$
\mu_{T}(A)=0 \quad \text { or } \quad \mu_{T}(A)=\mu_{T}(T) .
$$

We say that $S_{\mu}$ is an ergodic solenoid.

Definition 2.4. Let $S$ be a $k$-solenoid. The solenoid $S$ is uniquely ergodic if it has a unique (up to scalars) transversal measure $\mu$ and its support is the whole of $S$.

Now let $M$ be a smooth manifold of dimension $n$. An immersion of a $k$-solenoid $S$ into $M$, with $k<n$, is a smooth map $f: S \rightarrow M$ such that the differential restricted to the tangent spaces of leaves has rank $k$ at every point of $S$. The solenoid $f: S \rightarrow M$ is transversally 
immersed if for any flow-box $U \subset S$ and chart $V \subset M$, the map $f: U=D^{k} \times K(U) \rightarrow V \subset \mathbb{R}^{n}$ is an embedding, and the images of the leaves intersect transversally in $M$. If moreover $f$ is injective, then we say that the solenoid is embedded.

Note that under a transversal immersion, resp. an embedding, $f: S \rightarrow M$, the images of the leaves are immersed, resp. injectively immersed, submanifolds.

Definition 2.5. (Generalized currents) Let $S$ be an oriented $k$-solenoid of class $^{r, s}, r \geq 1$, endowed with a transversal measure $\mu=\left(\mu_{T}\right)$. An immersion

$$
f: S \rightarrow M
$$

defines a current $\left(f, S_{\mu}\right) \in \mathcal{C}_{k}(M)$, called generalized Ruelle-Sullivan current (or just generalized current), as follows. Let $\omega$ be an $k$-differential form in $M$. The pull-back $f^{*} \omega$ defines a $k$-differential form on the leaves of $S$. Let $S=\bigcup_{i} S_{i}$ be a measurable partition such that each $S_{i}$ is contained in a flow-box $U_{i}$. We define

$$
\left\langle\left(f, S_{\mu}\right), \omega\right\rangle=\sum_{i} \int_{K\left(U_{i}\right)}\left(\int_{L_{y} \cap S_{i}} f^{*} \omega\right) d \mu_{K\left(U_{i}\right)}(y),
$$

where $L_{y}$ denotes the horizontal disk of the flow-box.

The current $\left(f, S_{\mu}\right)$ is closed, hence it defines a real homology class

$$
\left[f, S_{\mu}\right] \in H_{k}(M, \mathbb{R}),
$$

called Ruelle-Sullivan homology class.

Note that this definition does not depend on the measurable partition (given two partitions consider the common refinement). If the support of $f^{*} \omega$ is contained in a flow-box $U$ then

$$
\left\langle\left(f, S_{\mu}\right), \omega\right\rangle=\int_{K(U)}\left(\int_{L_{y}} f^{*} \omega\right) d \mu_{K(U)}(y) .
$$

In general, take a partition of unity $\left\{\rho_{i}\right\}$ subordinated to the covering $\left\{U_{i}\right\}$, then

$$
\left\langle\left(f, S_{\mu}\right), \omega\right\rangle=\sum_{i} \int_{K\left(U_{i}\right)}\left(\int_{L_{y}} \rho_{i} f^{*} \omega\right) d \mu_{K\left(U_{i}\right)}(y) .
$$

Let us see that $\left(f, S_{\mu}\right)$ is closed. For any exact differential $\omega=d \alpha$ we have

$$
\begin{aligned}
\left\langle\left(f, S_{\mu}\right), d \alpha\right\rangle= & \sum_{i} \int_{K\left(U_{i}\right)}\left(\int_{L_{y}} \rho_{i} f^{*} d \alpha\right) d \mu_{K\left(U_{i}\right)}(y) \\
= & \sum_{i} \int_{K\left(U_{i}\right)}\left(\int_{L_{y}} d\left(\rho_{i} f^{*} \alpha\right)\right) d \mu_{K\left(U_{i}\right)}(y) \\
& \quad-\sum_{i} \int_{K\left(U_{i}\right)}\left(\int_{L_{y}} d \rho_{i} \wedge f^{*} \alpha\right) d \mu_{K\left(U_{i}\right)}(y)=0 .
\end{aligned}
$$

The first term vanishes using Stokes in each leaf (the form $\rho_{i} f^{*} \alpha$ is compactly supported on $\left.U_{i}\right)$, and the second term vanishes because $\sum_{i} d \rho_{i} \equiv 0$. Therefore $\left[f, S_{\mu}\right]$ is a well defined homology class of degree $k$.

In their original article $\mathrm{RuSu}$, Ruelle and Sullivan defined this notion for the restricted class of solenoids embedded in $M$. 
When $M$ is a compact and oriented $n$-manifold, the Ruelle-Sullivan homology class $\left[f, S_{\mu}\right] \in$ $H_{k}(M, \mathbb{R})$ gives an element

$$
\left[f, S_{\mu}\right]^{*} \in H^{n-k}(M, \mathbb{R}),
$$

under the Poincaré duality isomorphism $H_{k}(M, \mathbb{R}) \cong H^{n-k}(M, \mathbb{R})$.

We have the following result (theorem 7.6 in [MPM2]) which proves Proposition 1.2,

Theorem 2.6. (Self-intersection of embedded solenoids) Let $M$ be a compact, oriented, smooth manifold. Let $f: S_{\mu} \rightarrow M$ be an embedded oriented measured solenoid, such that the transversal measures $\left(\mu_{T}\right)$ have no atoms. Then we have

$$
\left[f, S_{\mu}\right]^{*} \cup\left[f, S_{\mu}\right]^{*}=0
$$

in $H^{2(n-k)}(M, \mathbb{R})$.

This indicates that we cannot use only embedded solenoids to represent real homology classes in general.

Now let us recall the notions of Schwartzman theory that we are going to need, and that are extensively studied in MPM3].

Let $M$ be a compact smooth Riemannian manifold. Given an Riemannian immersion $c$ : $N \rightarrow M$ from an oriented complete smooth manifold $N$ of dimension $k \geq 1$, we consider exhaustions $\left(U_{n}\right)$ of $N$ with $U_{n} \subset N$ being $k$-dimensional compact submanifolds with boundary $\partial U_{n}$. We close $U_{n}$ with a $k$-dimensional oriented manifold $\Gamma_{n}$ with boundary $\partial \Gamma_{n}=-\partial U_{n}$ (that is, $\partial U_{n}$ with opposite orientation, so that $N_{n}=U_{n} \cup \Gamma_{n}$ is a $k$-dimensional compact oriented manifold without boundary), in such a way that $c_{\mid U_{n}}$ extends to a piecewise smooth map $c_{n}: N_{n} \rightarrow M$. We may consider the associated homology class $\left[c_{n}\left(N_{n}\right)\right] \in H_{k}(M, \mathbb{Z})$. Suppose that

If the following limit exists,

$$
\frac{\operatorname{Vol}_{k}\left(c_{n}\left(\Gamma_{n}\right)\right)}{\operatorname{Vol}_{k}\left(c_{n}\left(N_{n}\right)\right)} \rightarrow 0
$$

$$
\lim _{n \rightarrow+\infty} \frac{1}{\operatorname{Vol}_{k}\left(c_{n}\left(N_{n}\right)\right)}\left[c_{n}\left(N_{n}\right)\right] \in H_{k}(M, \mathbb{R}),
$$

we call it a Schwartzman asymptotic $k$-cycle.

Definition 2.7. The immersed manifold $c: N \rightarrow M$ represents a homology class $a \in H_{k}(M, \mathbb{R})$ if for all exhaustions $\left(U_{n}\right)$, the class (2) exists and equals a. We denote $[c, N]=a$, and call it the Schwartzman homology class of $(c, N)$.

For immersed solenoids $f: S \rightarrow M$, we may consider the Schwartzman homology classes associated to its leaves.

Definition 2.8. (Schwartzman representation of homology classes) Let $f: S_{\mu} \rightarrow M$ be an immersion in $M$ of an oriented measured $k$-solenoid $S$, and give $S$ the induced Riemannian structure. The immersed solenoid $f: S_{\mu} \rightarrow M$ fully represents a homology class a $\in H_{1}(M, \mathbb{R})$ if for all leaves $l \subset S$, we have that $(f, l)$ is a Schwartzman asymptotic $k$-cycle with $[f, l]=a$.

A class of solenoids with good properties are those which have a trapping region, since for them the holonomy is represented by a single map. The definition is cumbersome but very natural.

Definition 2.9. (Trapping region) An open subset $W \subset S$ of a solenoid $S$ is a trapping region if there exists a continuous map $\pi: S \rightarrow \mathbb{T}$ such that 
(1) For some $0<\epsilon_{0}<1 / 2, W=\pi^{-1}\left(\left(-\epsilon_{0}, \epsilon_{0}\right)\right)$.

(2) There is a global transversal $T \subset \pi^{-1}(\{0\})$.

(3) Each connected component of $\pi^{-1}(\{0\})$ intersects $T$ in exactly one point.

(4) 0 is a regular value for $\pi$.

(5) For each connected component $L$ of $\pi^{-1}(\mathbb{T}-\{0\})$ we have $\bar{L} \cap T=\{x, y\}$, where $\{x\} \in \bar{L} \cap T \cap \pi^{-1}\left(\left(-\epsilon_{0}, 0\right]\right)$ and $\{y\} \in \bar{L} \cap T \cap \pi^{-1}\left(\left[0, \epsilon_{0}\right)\right)$.

The main result of [MPM3] is the following theorem.

Theorem 2.10. Let $S$ be a minimal oriented $k$-solenoid endowed with a transversal uniquely ergodic measure $\mu \in \mathcal{M}_{\mathcal{L}}(S)$ and with a trapping region $W \subset S$. Consider an immersion $f: S \rightarrow M$ such that $f(W)$ is contained in a contractible ball in $M$. Then $f: S_{\mu} \rightarrow M$ fully represents its Ruelle-Sullivan homology class $\left[f, S_{\mu}\right]$.

\section{Realization of $H_{1}(M, \mathbb{R})$}

Let $M$ be a $C^{\infty}$ smooth compact Riemannian manifold. Given a real 1-homology class $a \in H_{1}(M, \mathbb{R})$, we want to construct an immersion $f: S \rightarrow M$ in $M$ of a uniquely ergodic solenoid $S_{\mu}$ with generalized current $\left[f, S_{\mu}\right]=a$.

In some situations (depending on the dimension) we will achieve an embedding. Actually the abstract 1-solenoid $S$ that we will construct is independent of $a$ and of $M$, and moreover it has a 1-dimensional transversal structure.

Let $h: \mathbb{T} \rightarrow \mathbb{T}$ be a diffeomorphism of the circle with an irrational rotation number (and therefore uniquely ergodic), which is a Denjoy counter-example, i.e. has the unique invariant probability measure supported in the Cantor set $K \subset \mathbb{T}$. Let $\mu_{K}$ denote the invariant probability measure. For the original construction of Denjoy counter-examples see [De]. Actually for any given $\epsilon>0, h$ can be taken to be of class $C^{2-\epsilon}$ (see $[\mathrm{Her}$ ).

The suspension of $h$,

$$
S_{h}=([0,1] \times \mathbb{T}) /(0, x) \sim(1, h(x))
$$

is $C^{2-\epsilon}$-diffeomorphic to the 2-torus $T^{2}$. More explicitly, the diffeomorphism is as follows: take $c>0$ small, let $h_{t}, t \in[0, c]$, be a (smooth) isotopy from id to $h$, then we define the diffeomorphism $H: T^{2} \rightarrow S_{h}$ by

$$
H(t, x)= \begin{cases}\left(t, h^{-1}\left(h_{t}(x)\right)\right), & \text { for } t \in[0, c], \\ (t, x), & \text { for } t \in[c, 1] .\end{cases}
$$

Note that $S_{h}$ is foliated by the horizontal leaves, so $T^{2}$ is foliated accordingly. It can be considered also as a 1-solenoid of class $C^{\omega, 2-\epsilon}$.

The sub-solenoid

$$
S=([0,1] \times K) / \sim \subset S_{h}
$$

is an oriented 1-solenoid of class $C^{\omega, 2-\epsilon}$, with transversal $T=(\{0\} \times \mathbb{T}) \cap S=\{0\} \times K$. The holonomy is given by the map $h$, which is uniquely ergodic. Moreover, the associated transversal measure is $\mu_{K}$ on the transversal $K \cong\{0\} \times K$. So $S$ is an oriented and uniquely ergodic 1-solenoid.

Using the diffeomorphism $H$, we may see the solenoid $S$ inside the 2-torus, $S \subset S_{h} \cong T^{2}$, consisting of the paths $(t, x), x \in K, t \in[c, 1]$, together with the paths $\left(t, h_{t}(x)\right), x \in K$, $t \in[0, c]$. The embedding $S \hookrightarrow T^{2}$ is of class $C^{\omega, 2-\epsilon}$, so we shall think of $S$ as an oriented 1-solenoid of regularity $C^{\omega, 2-\epsilon}$. 


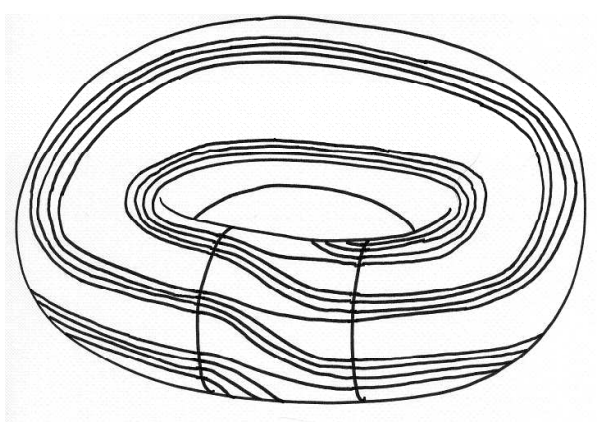

Figure 1. The 1-solenoid $S$.

Theorem 3.1. Let $M$ be a compact smooth manifold, and let $a \in H_{1}(M, \mathbb{R})$ be a non-zero 1-homology class. If $\operatorname{dim} M \geq 3$ then (a positive multiple of) a can be fully represented by an embedding (of class $C^{\infty, 2-\epsilon}$ ) of the (oriented, uniquely ergodic) 1-solenoid $S$ into $M$. If $\operatorname{dim} M=2$ then (a positive multiple of) a can be fully represented by a transversal immersion of $S$ into $M$.

Proof. Let $C_{1}, \ldots, C_{b_{1}}$ be (integral) 1-cycles which form a basis of the (real) 1-homology of $M$. Switch orientations and reorder the cycles if necessary so that there are real numbers $\lambda_{1}, \ldots, \lambda_{r}>0$ such that

$$
a=\lambda_{1} C_{1}+\cdots+\lambda_{r} C_{r} .
$$

By dividing by $\sum \lambda_{i}$ if necessary, we can assume that $\sum \lambda_{i}=1$.

Consider the solenoid $S$ constructed above and partition the cantor set $K$ into $r$ disjoint compact subsets $K_{1}, \ldots, K_{r}$ in cyclic order, each of which with

$$
\mu_{K}\left(K_{i}\right)=\lambda_{i} .
$$

Consider the transversal $T=\{0\} \times \mathbb{T}$ in $S_{h}$. We consider angles $\theta_{1}, \theta_{2}, \ldots, \theta_{n} \in \mathbb{T}$ in the same cyclic order as the $K_{i}$, such that $K_{i}$ is contained in the open subset $U_{i} \subset T$ with boundary points $\theta_{i}$ and $\theta_{i+1}$ (denoting $\theta_{n+1}=\theta_{1}$ ). We may assume that $\theta_{1}=0$. Remove the segments $[c, 1] \times\left\{\theta_{i}\right\}$ from $S_{h}$ to get the open 2-manifold

$$
U=S_{h}-\cup_{i}\left([c, 1] \times\left\{\theta_{i}\right\}\right) .
$$

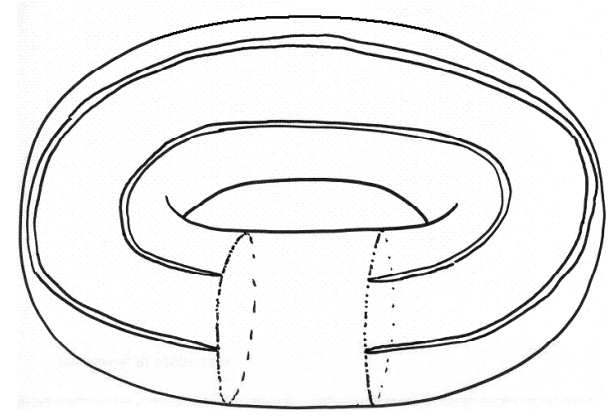

Figure 2. The open manifold $U$.

By construction, our solenoid $S$ is included as a subset of $U, S \subset U$.

Suppose that $\operatorname{dim} M \geq 3$. Then we can $C^{\infty}$-smoothly embed $F: U \rightarrow M$ as follows: suppose that all cycles $C_{i}$ share a common base-point $p_{0} \in M$ (and are otherwise disjoint to each other). 
Then embed the central part $(0, c) \times \mathbb{T} \subset U$ in a small ball $B$ around $p_{0}$ and embed each of the $[c, 1] \times U_{i}$ in $M-B$ in such a way that if we contract $B$ to $p_{0}$ then the images of $[c, 1] \times\{t\}$, $t \in U_{i}$, represent cycles homologous to $C_{i}$.

The embedding $f$ of $S$ into $M$ is defined as the composition $S \hookrightarrow U \stackrel{F}{\rightarrow} M$. By theorem 2.10, as $S$ is uniquely ergodic, to prove that $f: S \rightarrow M$ fully represents $a$, it is enough to see that $\left[f, S_{\mu}\right]=a$.

Let $\alpha$ be any closed 1-form on $M$. Since $H^{1}(M)=H^{1}(M, B)$, we may assume that $\alpha$ vanishes on $B$. We cover the solenoid $S$ by the flow-boxes $((0, c) \times \mathbb{T}) \cap S$ and $[c, 1] \times K_{i}$, $i=1, \ldots, r$. As $f^{*} \alpha$ vanishes in the first flow-box, we have

$$
\begin{aligned}
\left\langle\left[f, S_{\mu}\right],[\alpha]\right\rangle & =\sum_{i=1}^{r} \int_{K_{i}}\left(\int_{[c, 1]} f^{*} \alpha\right) d \mu_{K_{i}}(y)=\sum_{i=1}^{r} \int_{K_{i}}\left\langle C_{i},[\alpha]\right\rangle d \mu_{K_{i}}(y) \\
& =\sum_{i=1}^{r}\left\langle C_{i},[\alpha]\right\rangle \mu\left(K_{i}\right)=\sum_{i=1}^{r} \lambda_{i}\left\langle C_{i},[\alpha]\right\rangle=\langle a,[\alpha]\rangle
\end{aligned}
$$

proving that $\left[f, S_{\mu}\right]=a$.

Now suppose that $\operatorname{dim} M=2$. Let us do the appropriate modifications to the previous construction. Choose cycles $C_{i}$ sharing a common base-point $p_{0} \in M$, and such that their intersections (and self-intersections) away from $p_{0}$ are transversal. Changing $C_{i}$ by $2 C_{i}$ if necessary, we suppose that going around $C_{i}$ does not change the orientation (that is, the normal bundle to $C_{i}$ is oriented, hence trivial). From the manifold $U$ in Figure 2, remove $[0, c] \times\left\{\tau_{1}\right\}$ to get the open 2-manifold

$$
V=((0, c) \times(0,1)) \bigcup \cup_{i}\left([c, 1] \times U_{i}\right) .
$$

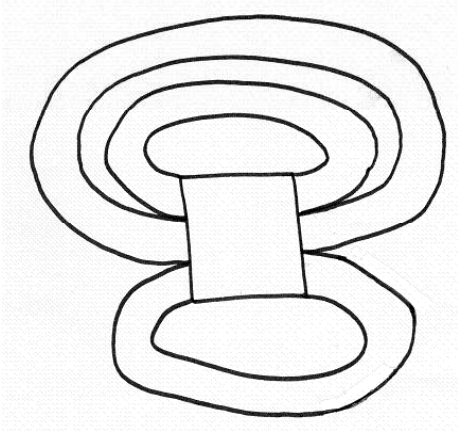

FiguRE 3. The open manifold $V$

The manifold $V$ can be immersed into the surface $M, F: V \rightarrow M$, in such a way that $(0, c) \times(0,1)$ is sent to a ball $B$ around $p_{0},[c, 1] \times U_{i}$ are sent to $M-B$, the images of $[c, 1] \times\{t\}, t \in U_{i}$, represent cycles homologous to $C_{i}$ if we contract $B$ to a point, and the intersections and self-intersections of horizontal leaves are always transverse.

Note that the solenoid $S$ is not contained in $V$, since we have removed $[0, c] \times \tau_{1}$ from $U$. So we cannot define an immersion $f: S \rightarrow M$ by restricting that of $F$. To define $f$ in $S \cap((0, c) \times \mathbb{T})$, we need to explicit out our isotopy $h_{t}$. Consider $h: \mathbb{T} \rightarrow \mathbb{T}$ and lift it to $\tilde{h}: \mathbb{R} \rightarrow \mathbb{R}$ with $r:=\tilde{h}(0) \in(0,1)$. Consider a smooth function $\rho: \mathbb{R} \rightarrow[0,1]$, with $\rho(t)=1$ for 
$t \leq 0, \rho(t)=0$ for $t \geq c$, and $\rho^{\prime}(t)<0$ for $t \in(0, c)$. Then we can define

$$
h_{t}(x)=\tilde{h}\left(\tilde{h}^{-1}(x) \rho(t)+x(1-\rho(t))\right) \quad \bmod \mathbb{Z} .
$$

Define the immersion $f: S \rightarrow M$ as follows: $f$ equals $F$ for $(t, x) \in[c, 1] \times K \subset V$. For $\left(t, h^{-1}\left(h_{t}(x)\right)\right) \in S \cap([0, c] \times \mathbb{T})$, we set

$$
f\left(t, h^{-1}\left(h_{t}(x)\right)\right)= \begin{cases}F\left(t,\left(\tilde{h}^{-1}(x)+1\right) \rho(t)+x(1-\rho(t))\right), & x \in K \cap(0, r), \\ F\left(t, \tilde{h}^{-1}(x) \rho(t)+x(1-\rho(t))\right), & x \in K \cap(r, 1) .\end{cases}
$$

It is easily checked that $f$ sends $S \cap([0, c] \times \mathbb{T})$ into the ball $B$ and the intersections of the leaves in this portion of the solenoid are transverse.

The proof that the Ruelle-Sullivan homology class of $f: S \rightarrow M$ is $\left[f, S_{\mu}\right]=a$ goes as before.

Remark 3.2. We do not need $M$ to be compact for the above construction to work. If $M$ is non-compact, take integer 1-cycles $C_{1}, C_{2}, \ldots$ (possibly infinitely many) which form a basis of $H_{1}(M, \mathbb{R})$. Then for any $a \in H_{1}(M, \mathbb{R})$ there exist an integer $r \geq 1$ and $\lambda_{1}, \ldots, \lambda_{r} \in \mathbb{R}$ with $a=\sum \lambda_{i} C_{i}$. The construction of theorem 3.1 works.

The solenoid $S$ is oriented, regardless of $M$ being oriented or not.

\section{Realization of $H_{k}(M, \mathbb{R})$}

Let $M$ be a smooth compact oriented Riemannian $C^{\infty}$ manifold and let $a \in H_{k}(M, \mathbb{R})$ be a non-zero real $k$-homology class. We are going to construct a uniquely ergodic $k$-solenoid $f: S \rightarrow M$ with a 1-dimensional transversal structure, immersed in $M$ and fully representing $a$.

To start with, fix a collection of compact $k$-dimensional smooth oriented manifolds $S_{1}, \ldots, S_{r}$ and positive numbers $\lambda_{1}, \ldots, \lambda_{r}>0$ such that $\sum \lambda_{i}=1$. For any fixed $\epsilon>0$, let $h: \mathbb{T} \rightarrow \mathbb{T}$ be a diffeomorphism of the circle which is a Denjoy counter-example with an irrational rotation number and of class $C^{2-\epsilon}$. Hence $h$ is uniquely ergodic. Let $\mu_{K}$ be the unique invariant probability measure, which is supported in the minimal Cantor set $K \subset \mathbb{T}$. Partition the Cantor set $K$ into $r$ disjoint compact subsets $K_{1}, \ldots, K_{r}$ in cyclic order, each of which with $\mu_{K}\left(K_{i}\right)=\lambda_{i}$.

We fix two points on each manifold $S_{i}$, and remove two small balls, $D_{i}^{+}$and $D_{i}^{-}$, around them. Denote

$$
S_{i}^{\prime}=S_{i}-\left(D_{i}^{+} \cup D_{i}^{-}\right),
$$

so that $S_{i}^{\prime}$ is a manifold with oriented boundary $\partial S_{i}^{\prime}=\partial D_{i}^{+} \sqcup \partial D_{i}^{-}$. Fix two diffeomorphisms: $\partial D_{i}^{+} \cong S^{k-1}$, being orientation preserving, and $\partial D_{i}^{-} \cong S^{k-1}$, being orientation reversing. There are inclusions

$$
A_{ \pm}:=\bigsqcup\left(\partial D_{i}^{ \pm} \times K_{i}\right) \stackrel{i_{ \pm}}{\hookrightarrow} S^{k-1} \times S^{1},
$$

whose image is $S^{k-1} \times K \subset S^{k-1} \times S^{1}$. Define

$$
S=\bigsqcup\left(S_{i}^{\prime} \times K_{i}\right)_{/(x, y) \sim i_{+}^{-1} \circ(\mathrm{id} \times h) \circ i_{-}(x, y),(x, y) \in A_{-}} .
$$

This is an oriented $k$-solenoid of class $C^{\infty, 2-\epsilon}$, with 1-dimensional transversal dimension. As $S^{k-1} \times K \subset S$ in an obvious way, fixing a point $p \in S^{k-1}$, we have a global transversal $T=\{p\} \times K \subset S^{k-1} \times K \subset S$. Identifying $T \cong K$, the holonomy pseudo-group is generated by $h: K \rightarrow K$. Hence $S$ is uniquely ergodic. Let $\mu$ denote the transversal measure corresponding to $\mu_{K}$. 
We want to give an alternative description of $S$. Fix an isotopy $h_{t}, t \in[0,1]$, from id to $h$. Define the set

$$
W^{\prime}:=\left\{\left(t, x, h^{-1}\left(h_{t}(y)\right)\right) ; t \in[0,1], x \in S^{k-1}, y \in K\right\} \subset[0,1] \times S^{k-1} \times S^{1} .
$$

Then we have that

$$
S=\left(\bigsqcup\left(S_{i}^{\prime} \times K_{i}\right) \sqcup W^{\prime}\right)_{\substack{(x, y) \sim\left(0, i_{-}(x, y)\right),(x, y) \in \partial D_{i}^{-} \times K_{i} \\(x, y) \sim\left(1, i_{+}(x, y)\right),(x, y) \in \partial D_{i}^{+} \times K_{i}}} .
$$

Strictly speaking, we should say that they are diffeomorphic, but we shall fix an identification. We define a map $\pi: S \rightarrow \mathbb{T}$ by

$$
\begin{cases}\pi\left(t, x, h^{-1}\left(h_{t}(y)\right)\right)=t-\frac{1}{2}, & \left(t, x, h^{-1}\left(h_{t}(y)\right)\right) \in W^{\prime}, \\ \pi(p)=\frac{1}{2}, & p \in S-W^{\prime} .\end{cases}
$$

Then $W=\operatorname{Int}\left(W^{\prime}\right)=\pi^{-1}\left(-\frac{1}{2}, \frac{1}{2}\right)$ is a trapping region according to definition 2.9.

Consider angles $\tau_{1}, \tau_{2}, \ldots, \tau_{n} \in \mathbb{T}$ in the same cyclic order as the $K_{i}$, such that $K_{i}$ is contained in the open subset $U_{i} \subset T$ with boundary points $\tau_{i}$ and $\tau_{i+1}$ (denoting $\tau_{n+1}=\tau_{1}$ ). We may assume that $\tau_{1}=0$. Then the solenoid $S$ sits inside the $(k+1)$-dimensional open manifold

$$
X=\bigsqcup\left(S_{i}^{\prime} \times U_{i}\right) \sqcup\left([0,1] \times S^{k-1} \times S^{1}\right)_{\substack{(x, y) \sim\left(0, i_{-}(x, y)\right),(x, y) \in \partial D_{i}^{-} \times U_{i} \\(x, y) \sim\left(1, i_{+}(x, y)\right),(x, y) \in \partial D_{i}^{+} \times U_{i}}},
$$

as the collection of points $(x, y), x \in S_{i}^{\prime}, y \in K_{i}$, together with the points $\left(t, x, h^{-1}\left(h_{t}(y)\right)\right)$, $x \in S^{k-1}, y \in K, t \in[0,1]$.

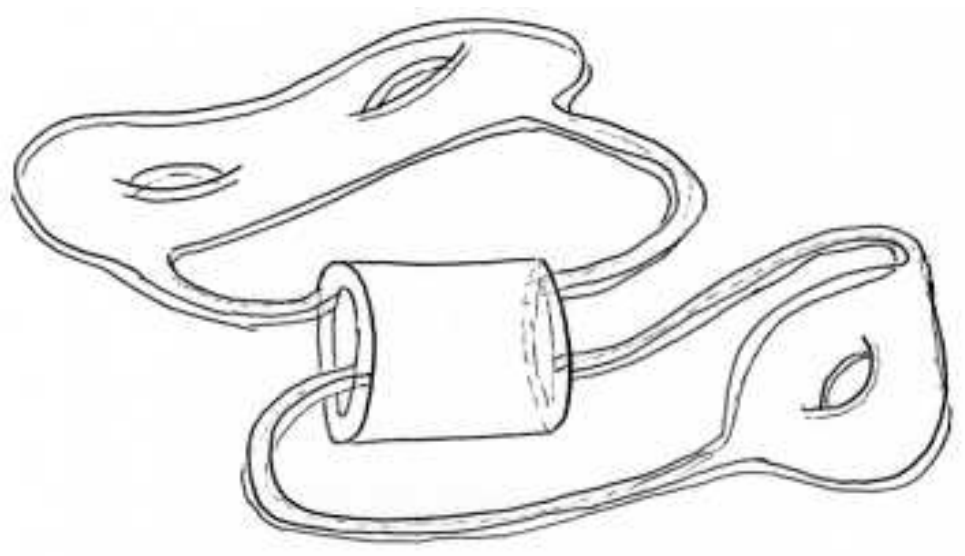

Figure 4. The manifold $X$.

Remark 4.1. The 1-solenoid constructed in section 3 corresponds to the case $S_{i}=S^{1}, i=$ $1, \ldots, r$.

Theorem 4.2. Let $M$ be a compact oriented smooth Riemannian manifold of dimension $n$, and let $a \in H_{k}(M, \mathbb{R})$ be a non-zero real k-homology class. Then (a positive multiple of) a can be fully represented by a transversal immersion $f: S \rightarrow M$ of a uniquely ergodic oriented $k$-solenoid. If moreover, $n \geq 2 k+1$ then we can suppose that $f$ is an embedding. 


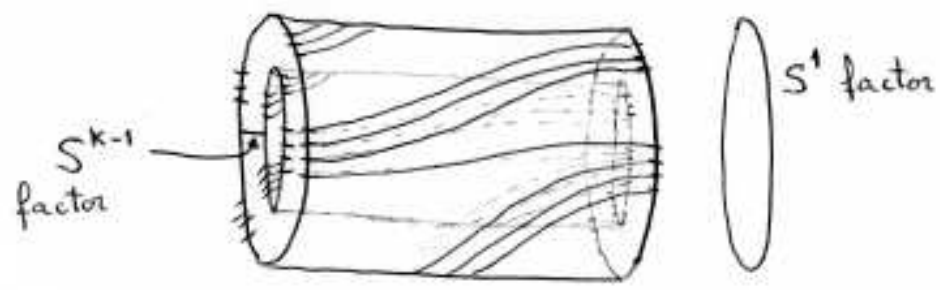

Figure 5. The trapping region $W^{\prime}$.

Proof. By proposition A.3, we may take a collection $C_{1}, \ldots, C_{b_{k}} \in H_{k}(M, \mathbb{Z})$ which are a basis of $H_{k}(M, \mathbb{Q})$ and such that $C_{i}$ are represented by immersed submanifolds $S_{i} \subset M$ with trivial normal bundle and self-transverse intersections, and such that $S_{i}$ intersects $S_{j}$ transversally. Moreover, if $n \geq 2 k+1$, we may assume that there are no either intersections or self-intersections.

After switching the orientations of $C_{i}$ if necessary, reordering the cycles and multiplying $a$ by a suitable positive real number, we may suppose that

$$
a=\lambda_{1} C_{1}+\ldots+\lambda_{r} C_{r}
$$

for some $r \geq 1, \lambda_{i}>0,1 \leq i \leq r$, and $\sum \lambda_{i}=1$. We construct the solenoid $S$ with the procedure above starting with the manifolds $S_{i}$ and coefficients $\lambda_{i}$. This is a uniquely ergodic $k$-solenoid with a 1-dimensional transversal structure, and a trapping region $W \subset S$ (see [MPM3].

Now we want to define an immersion $f: S \rightarrow M$, and to prove that it fully represents $a$. We have the following cases:

(1) $n \geq 2 k+1$. The general position property on the $S_{i}$ implies that all $S_{i}$ are disjoint submanifolds of $M$. As the normal bundle to $S_{i}$ is trivial and $U_{i}$ is an interval, we can embedded $S_{i} \times U_{i}$ in a small neighbourhood of $S_{i}$.

Fix a base point $p_{0} \in M$ off all $S_{i}$. Take a small box $B \subset M$ around $p_{0}$ of the form $B=[0,1] \times D^{n-1}$, where $D^{n-1}$ is the open $(n-1)$-dimensional ball. Consider a circle $S^{1} \subset D^{k+1} \subset D^{n-1}$ and let $D^{k} \times S^{1} \subset D^{k+1} \subset D^{n-1}$ be a tubular neighbourhood of it, with boundary $S^{k-1} \times S^{1}$.

For each $i=1, \ldots, r$, fix $y_{i} \in U_{i}$, and consider two paths in $M-\operatorname{Int}(B)$, say $\gamma_{i}^{ \pm}$, where $\gamma_{i}^{-}$goes from the point $\left(0, y_{i}\right) \in\{0\} \times U_{i} \subset\{0\} \times S^{1} \subset\{0\} \times D^{n-1} \subset B$ to the point $\left(p_{i}^{-}, y_{i}\right) \in S_{i} \times U_{i}$, and $\gamma_{i}^{+}$goes from $\left(1, y_{i}\right) \in\{1\} \times U_{i} \subset\{1\} \times S^{1} \subset\{1\} \times D^{n-1} \subset B$ to $\left(p_{i}^{+}, y_{i}\right) \in S_{i} \times U_{i}$. We arrange that $\gamma_{i}^{ \pm}$are transverse to $S_{i} \times U_{i}$ at $\left(p_{i}^{ \pm}, y_{i}\right)$ and are disjoint from all $S_{j}$ otherwise.

We thicken $\gamma_{i}^{ \pm}$to immersions $\gamma_{i}^{ \pm} \times D^{k} \times U_{i}$ into $M-\operatorname{Int}(B)$ such that one extreme goes to $D_{i}^{ \pm} \times U_{i}$ and the other goes to either $D^{k} \times U_{i} \times\{0\} \subset D^{k} \times S^{1} \times\{0\} \subset D^{n-1} \times\{0\} \subset B$ for $\gamma_{i}^{-}$, or $D^{k} \times U_{i} \times\{1\} \subset D^{k} \times S^{1} \times\{1\} \subset D^{n-1} \times\{1\} \subset B$ for $\gamma_{i}^{-}$. It is possible to do this in such a way that the $U_{i}$ directions match, since $n \geq k+2$.

Recall that $S_{i}^{\prime}=S_{i}-\left(D_{i}^{+} \cup D_{i}^{-}\right)$, and set

$$
S_{i}^{\prime \prime}=S_{i}^{\prime} \cup\left(\gamma_{i}^{+} \times S^{k-1}\right) \cup\left(\gamma_{i}^{-} \times S^{k-1}\right),
$$


which is diffeomorphic to $S_{i}^{\prime}$ (to be rigorous, we should smooth out corners). Then we can define the set

$$
\begin{aligned}
U:= & \bigcup\left(\left(S_{i}^{\prime} \times U_{i}\right) \cup\left(\gamma_{i}^{+} \times S^{k-1} \times U_{i}\right) \cup\right. \\
& \cup\left(\gamma_{i}^{-} \times S^{k-1} \times U_{i}\right) \cup\left([0,1] \times S^{k-1} \times S^{1}\right),
\end{aligned}
$$

which is a $(k+1)$-dimensional open manifold embedded in $M$. The manifold $U$ is foliated as follows: $S_{i}^{\prime \prime} \times U_{i}$ is foliated by $S_{i}^{\prime \prime} \times\{y\}$, for $y \in U_{i}$, and $[0,1] \times S^{k-1} \times S^{1}$ is foliated by

$$
L_{y}=\left\{\left(t, x, h^{-1}\left(h_{t}(y)\right)\right) ; t \in[0,1], x \in S^{k-1}\right\},
$$

for $y \in S^{1}$. Clearly the solenoid $S$ is a sub-solenoid of $U, S \subset U$. Restricting the embedding $F: U \rightarrow M$ to $S$ we get an embedding $f: S \rightarrow M$.

By construction $f(W) \subset \operatorname{Int}(B)$, i.e. the image of the trapping region is contained in a contractible ball.

(2) $1<n-k \leq k$. The same construction as in (1) works now, with the modification that we have to allow intersections of different leaves, but we may take them to be always transversal. So we get a transversal immersion $f: S \rightarrow M$.

(3) $n-k=1$. The submanifolds $S_{i}$ have trivial normal bundle and they intersect each other transversally. We cannot avoid that the paths $\gamma_{i}^{ \pm}$intersect other $S_{j}$, but we arrange these intersections to be transverse. This produces a transversal immersion $f$ of the region $S-W$ of the solenoid into $M-\operatorname{Int}(B)$.

We have to modify the previous construction of the immersion of $W$ into $B$, as codimension one does not leave enough room for it to work. Consider the box $B=$ $[0,1] \times D^{n-1}$ and remove the axis $A=[0,1] \times\{0\}$. Use polar coordinates to identify $B-A=[0,1] \times S^{k-1} \times(0,1)$, where the third coordinate corresponds to the radius. By construction, $W^{\prime} \subset S$ embeds into $C=[0,1] \times S^{k-1} \times S^{1}$, as the set of points $\left(t, x, h^{-1}\left(h_{t}(y)\right)\right), t \in[0,1], x \in S^{k-1}$ and $y \in K$. We remove $D=[0,1] \times S^{k-1} \times \tau_{1}$ from $C$, so that $C-D=[0,1] \times S^{k-1} \times(0,1)$. Then $W^{\prime}$ immerses into $C-D$, by using the process at the end of the proof of theorem 3.1 (now there is an extra factor $S^{k-1}$ which plays no role). This is a transversal immersion.

There is one extra detail that we should be careful about. When connecting $p_{i}^{ \pm}$with the two faces of $B$, the orientations of the $U_{i}$ should match. This happens because the normal bundle to $S_{i}$ is trivial, and in this case $S_{i} \times U_{i}$ is (diffeomorphic to) the normal bundle to $S_{i}$.

We prove now that $f: S \rightarrow M$ fully represents $a$, we use theorem 2.10. The solenoid $S$ has a trapping region $W$, and $f(W) \subset \operatorname{Int}(B)$, a contractible ball in $M$. So we only need to see that $\left[f, S_{\mu}\right]=a$.

Recall that the associated transversal measure is $\mu_{K}$ on the transversal $K$. Let $\alpha$ be any closed 1-form on $M$. Since $H^{1}(M)=H^{1}(M, B)$, we may assume that $\alpha$ vanishes on $B$. We cover the solenoid $S$ by the flow-boxes $S_{i}^{\prime \prime} \times K_{i}, i=1, \ldots, r$, and $W^{\prime}$ (where the form $\alpha$ vanishes). Thus

$$
\begin{aligned}
\left\langle\left[f, S_{\mu}\right],[\alpha]\right\rangle & =\sum_{i=1}^{r} \int_{K_{i}}\left(\int_{S_{i}^{\prime \prime}} f^{*} \alpha\right) d \mu_{K_{i}}(y)=\sum_{i=1}^{r} \int_{K_{i}}\left\langle C_{i},[\alpha]\right\rangle d \mu_{K_{i}}(y) \\
& =\sum_{i=1}^{r}\left\langle C_{i},[\alpha]\right\rangle \mu\left(K_{i}\right)=\sum_{i=1}^{r} \lambda_{i}\left\langle C_{i},[\alpha]\right\rangle=\langle a,[\alpha]\rangle
\end{aligned}
$$

proving that $\left[f, S_{\mu}\right]=a$. 
Remark 4.3. A similar comment to that of remark 3.2 applies to the present situation, that is, the compactness of $M$ is not necessary.

Remark 4.4. The orientability of $M$ is not necessary as well. If $M$ is non-orientable, we may consider its oriented double cover $\pi: \tilde{M} \rightarrow M$. Then for $a \in H_{k}(M, \mathbb{R})$, there exists $\tilde{a} \in H_{k}(\tilde{M}, \mathbb{R})$ with $\pi_{*}(\tilde{a})=a$.

We can consider immersed submanifolds $f_{i}: S_{i} \hookrightarrow \tilde{M}$ with transversal self-intersections, and intersecting transversally each other. Then it is easy to perturb $f_{i}$ so that $\tilde{f}_{i}=\pi \circ f_{i}: S_{i} \rightarrow$ $M$ are immersed oriented submanifolds with transversal self-intersections, and intersecting transversally each other. This will allow to construct a uniquely-ergodic oriented $k$-solenoid $f: S \rightarrow M$ transversally immersed in $\tilde{M}$ fully representing (a multiple of) $\tilde{a}$ such that $\pi \circ f$ : $S \rightarrow M$ is transversally immersed in $M$ and fully represents (a multiple of) $a$.

If $n \geq 2 k+1$, then we can assume that $f$ is an embedding (since transversal intersections in this dimension do not happen).

Remark 4.5. In the article [MPM1, we prove that the currents that we have constructed are general enough in order to fill a dense subset of the space of currents. Therefore, the generalized Ruelle-Sullivan currents associated to immersed measured oriented uniquely-ergodic solenoids are dense in the space of closed currents. This question was prompted to the authors by Dennis Sullivan.

\section{Appendix. Homology Classes RePResented By SUbmanifolds}

By a theorem of Thom (see [Th1] and [Th2]), if $a \in H_{k}(M, \mathbb{Z})$ then there exists $N>>1$ such that $N \cdot a$ is represented by a smooth submanifold of $M$. This submanifold $C \subset M$ is oriented because it represents a non-zero homology class (the top homology of a compact connected non-orientable manifold is zero). Moreover, if $n \geq 2 k+1$ or $n-k$ is odd then it can be arranged that the normal bundle of $C$ is trivial Th1 [Th2. If $n-k$ is even then it can be arranged that the normal bundle is trivial if and only if $a \cup a=0$. Also according to Sullivan Su2, using Thom's method and the thesis of Wells We one can always represent $N \cdot a$ by an immersed submanifold $f: C \rightarrow M$ with trivial normal bundle. (Note that the normal bundle is defined for any immersed manifold.) Moreover, with a small perturbation, we may assume that $f$ has only transversal self-intersections.

For completeness, we give here a proof of these results by elementary methods. We start first with the case of odd codimension.

Lemma A.1. Let $M$ be a compact and oriented manifold of dimension $n$. Let $0 \leq k \leq n$ with $n-k$ odd and $a \in H_{k}(M, \mathbb{Z})$.

There exists $N>>1$ (dependent only on $n$ and $k$ ) and a smooth map $f: M \rightarrow S^{n-k}$ such that for a generic point $p \in S^{n-k}$,

$$
C=f^{-1}(p) \subset M
$$

is a smooth submanifold with trivial normal bundle such that $[C]=N \cdot a$.

Proof. Let $\hat{a} \in H^{n-k}(M, \mathbb{Z})$ be the Poincaré dual of $a$. We aim to construct a map $f: M \rightarrow$ $S^{n-k}$ such that $f^{*}\left(\left[S^{n-k}\right]\right)$ is a multiple of $\hat{a}$. For this, consider a CW decomposition of $M$, and let $\bar{a} \in C^{n-k}(M, \mathbb{Z})$ with $\partial \bar{a}=0$ and $[\bar{a}]=\hat{a}$. 
We start by considering a map $f$ from the $(n-k-1)$-skeleton of $\bar{a}$ to a base point $p \in S^{n-k}$. To define $f$ in the $(n-k)$-skeleton, write

$$
\hat{a}=\sum_{i} n_{i} C_{i}^{*}
$$

with $C_{i}$ being the $(n-k)$-cells of $M$. Then define $f_{\mid C_{i}}$ in such a way that the induced map $f_{\mid C_{i}}: C_{i} / \partial C_{i} \rightarrow S^{n-k}$ has degree $n_{i}$.

To extend $f$ to the higher skeleta, we work as follows: let $T$ be an $(n-k+1)$-cell of $M$. Since

$$
\hat{a}(\partial T)=\partial \hat{a}(T)=0,
$$

we have that $f_{\mid \partial T}: \partial T \rightarrow S^{n-k}$ has degree 0 . Therefore, we can extend $f$ to a map $T \rightarrow$ $S^{n-k}$. Now by induction on $l=1,2, \ldots$ we assume that the map $f$ has been extended to the $(n-k+l-1)$-skeleton of $M$ and we wish to extend it to the $(n-k+l)$-skeleton. Let $T$ be a $(n-k+l)$-cell. The map $f_{\mid \partial T}: \partial T \rightarrow S^{n-k}$ gives, recalling that $\partial T \cong S^{n-k+l-1}$, an element

$$
\left[f_{\mid \partial T}\right] \in \pi_{n-k+l-1}\left(S^{n-k}\right) .
$$

By a result of Serre [Se], this group is torsion (since $n-k$ is odd). So there is a non-zero integer $k_{l}$ such that $k_{l} \cdot[\partial T]=0$. Multiplying $a$ by $m_{l}$, the map $f^{\prime}$ (in the $(n-k+l-1)$-skeleton) corresponding to $a^{\prime}=m_{l} \cdot a$ is the composition of $f$ with a map $S^{n-k} \rightarrow S^{n-k}$ of degree $k_{l}$. Therefore $\left[f_{\mid \partial T}^{\prime}\right]=m_{l} \cdot\left[f_{\mid \partial T}\right]=0$, and there is no obstruction to extend $f^{\prime}$ to the cell $T$, and hence to the $(n-k+l)$-skeleton.

In this way, we get an extension to the $n$-skeleton, i.e. to $M$. This gives a continuous map $f: M \rightarrow S^{n-k}$ and it is trivial to verify that $f^{*}\left(\left[S^{n-k}\right]\right)=N \cdot \hat{a}$, for some large integer $N$ (actually, $N=m_{2} m_{3} \cdots m_{k}$ ).

Now, we homotop $f$ to a smooth function, which we call $f$ again. Taking a regular value $p \in S^{n-k}$, we have a smooth submanifold $C=f^{-1}(p)$ of dimension $k$, and with trivial normal bundle. Clearly, $[C]=P D[N \cdot \hat{a}]=N \cdot a$.

Lemma A.2. Let $M$ be a compact and oriented manifold of dimension $n$. Let $1 \leq k \leq n$ with $n-k$ even and $a \in H_{k}(M, \mathbb{Z})$.

There exists $N>>1$ (only dependent of $n$ and $k$ ), an immersion $i: C \rightarrow M$ of an oriented compact manifold $C$ with $i_{*}[C]=N \cdot$ a and whose normal bundle $\nu_{C / M} \rightarrow C$ is trivial.

Proof. We consider $M \times \mathbb{R}$, which is an $(n+1)$-manifold. It is open, but the proof of lemma A.1 works for it and for the homology class $a \in H_{k}(M \times \mathbb{R}, \mathbb{Z}) \cong H_{k}(M, \mathbb{Z})$. Note that $(n+1)-k$ is odd, so lemma A.1 guarantees the existence of a smooth $k$-dimensional submanifold $C \subset M \times \mathbb{R}$ with trivial normal bundle, and such that $[C]=N \cdot a$, for some $N \geq 1$.

Denote by $j: C \hookrightarrow M \times \mathbb{R}$ the inclusion, and let $\pi: M \times \mathbb{R} \rightarrow M$ be the projection into the first factor. Denote by $t$ is the coordinate of the $\mathbb{R}$ direction, and by $\frac{\partial}{\partial t}$ the vertical vector field. Fixing a non-zero normal vector field $X$ to $C \subset M \times \mathbb{R}$, the compression theorem in RoSa] allows to isotop the pair $(j, X)$ to $\left(j^{\prime}, \frac{\partial}{\partial t}\right)$, where $j^{\prime}: C \hookrightarrow M \times \mathbb{R}$ is an embedding and $\frac{\partial}{\partial t}$ becomes a normal vector field to $j^{\prime}(C)$. Therefore the composition $i=\pi \circ j^{\prime}: C \rightarrow M$ is an immersion. Clearly, $i_{*}[C]=\pi_{*} j_{*}^{\prime}[C]=\pi_{*}[C]=\pi_{*}(N \cdot a)=N \cdot a \in H_{k}(M, \mathbb{R})$ and the normal bundle to $C$ in $M$ is trivial.

The precise result that we use in section 4 is the following: 
Proposition A.3. Let $M$ be a compact manifold of dimension $n$, and let $b_{k}=\operatorname{dim} H_{k}(M, \mathbb{R})$. Then we may take a collection $C_{1}, \ldots, C_{b_{k}} \in H_{k}(M, \mathbb{Z})$ which forms a basis of $H_{k}(M, \mathbb{Q})$ and such that $C_{i}$ are represented by immersed submanifolds $S_{i} \subset M$ with trivial normal bundle and self-transverse intersections, and such that $S_{i}$ intersects $S_{j}$ transversally. Moreover, if $n \geq 2 k+1$, we may assume that there are no either intersections or self-intersections.

Proof. Using lemma A.1 or lemma A.2 (according to the parity or $n-k$ ), we may find a collection of immersed oriented compact submanifolds $S_{i}$ with trivial normal bundle representing a basis for the rational homology $H_{k}(M, \mathbb{Q})$.

Now a small perturbation of each $S_{i}$ makes all intersections of $S_{i}$ with $S_{j}, i \neq j$, and all self-intersections of $S_{i}$, transverse. If $n \geq 2 k+1$, the transversality of the intersections implies that there are no intersections at all. So the result follows.

\section{REFERENCES}

[De] Denjoy, A. Sur les courbes définies par les équations différentielles à la surface du tore. J. Math. Pures et Appliquées (9. série) 11 (1932), 333-375.

[Her] Herman, M.R. Sur la conjugaison différentiable des difféomorphismes du cercle à des rotations. Inst. Hautes Études Sci. Publ. Math. 49 (1979), 5-233.

[HM] Hurder, S.; Mitsumatsu, Y. The intersection product of transverse invariant measures. Indiana Univ. Math. J. 40 (1991), no. 4, 1169-1183.

[MPM1] Muñoz, V.; Pérez-Marco, R. Ergodic solenoidal homology: Density of ergodic solenoids. Australian J. Math. Anal. and Appl. 6 (2009), no. 1, Article 11, 1-8.

[MPM2] Muñoz, V.; Pérez-Marco, R. Ergodic solenoids and generalized currents. Preprint.

[MPM3] Muñoz, V.; Pérez-Marco, R. Schwartzman cycles and ergodic solenoids. Preprint.

[RoSa] Rourke, C.; Sanderson, B. The compression theorem. Geometry \& Topology 5 (2001), 399-429.

[RuSu] Ruelle, D.; Sullivan, D. Currents, flows and diffeomorphisms. Topology 14 (1975), no. 4, 319-327.

[Sc] Schwartzman, S. Asymptotic cycles. Ann. of Math. (2) 66 (1957), 270-284.

[Se] Serre, J.-P. Groupes d'homotopie et classes de groupes abéliens. Ann. of Math. (2) 58 (1953), 258-294.

[Su1] Sullivan, D. Cycles for the dynamical study of foliated manifolds and complex manifolds. Invent. Math. 36 (1976), 225-255.

[Su2] Sullivan, D. René Thom's work on geometric homology class and bordism. Bulletin of the AMS, 41, 3, (2004), 341-350.

[Th1] Thom, R. Sous-variétés et classes d'homologie des variétés différentiables. I et II. C. R. Acad. Sci. Paris 236 (1953), 453-454 and 573-575.

[Th2] Thom, R. Quelques propriétés globales des variétés différentiables. Commentarii Mathematici Halvetici 236 (1954), 17-86.

[We] Wells, R. Cobordisms groups of immersions. Topology 5 (1966), 281-294.

[Zu] Zucker, S. The Hodge conjecture for cubic fourfolds. Compositio Math. 34 (1977), 199ï_-209. SPAIN

Instituto de Ciencias Matemáticas CSIC-UAM-UC3M-UCM, Serrano 113 Bis, 28006 Madrid,

Facultad de Matemáticas, Universidad Complutense de Madrid, Plaza de Ciencias 3,28040 MADRID, SPAIN

E-mail address: vicente.munoz@imaff.cfmac.csic.es

CNRS, LAGA UMR 7539, Université Paris XiII, 99, Avenue J.-B. Clément, 93430-Villetaneuse, France

E-mail address: ricardo@math.univ-paris13.fr 